\title{
University community-based survey on the knowledge, attitude and perception about COVID-19 pandemic: The Federal University of Agriculture, Abeokuta, Nigeria as a case study
}

Olubukola Tolulope ADENUBI ${ }^{a^{*}}$, Oluwawemimo Oluwaseun ADEBOWALE ${ }^{\mathrm{b}}$, Abimbola Adetokunbo OLOYE ${ }^{c}$, Noah Olumide Akorede BANKOLE ${ }^{\mathrm{d}}$, Patience Oluwatoyin AYOAJAYI $^{\mathrm{e}}$, Adebayo Koyuum AKINLOYE

a Department of Veterinary Pharmacology and Toxicology, College of Veterinary Medicine, Federal University of Agriculture, Abeokuta, Ogun State, Nigeria.

${ }^{b}$ Department of Veterinary Public Health and Preventive Medicine, College of Veterinary Medicine, Federal University of Agriculture, Abeokuta, Ogun State, Nigeria.

${ }^{\mathrm{c}}$ Department of Veterinary Surgery and Theriogenology, College of Veterinary Medicine, Federal University of Agriculture, Abeokuta, Ogun State, Nigeria.

${ }^{\mathrm{d}}$ Department of Veterinary Microbiology, College of Veterinary Medicine, Federal University of Agriculture, Abeokuta, Ogun State, Nigeria.

${ }^{\mathrm{e}}$ Nigerian Field Epidemiology and Laboratory Training Program.

${ }^{\mathrm{f}}$ Department of Veterinary Anatomy, College of Veterinary Medicine, Federal University of Agriculture, Abeokuta, Ogun State, Nigeria.

*Corresponding author: Olubukola Tolulope Adenubi, Tel: +2348025409691; E-mail: bukiadenubi@gmail.com; ORCID: 0000-0003-3445-8485

\section{Abstract}

The coronavirus disease (COVID-19), an emerging viral infection, is impacting the social, economic and political patterns of the world. To contain the spread of this pandemic, Nigeria like many countries globally, has imposed drastic preventive measures such as physical distancing and lockdown/curfew. This study assessed the knowledge, attitude and perception (KAP) about COVID-19 among members of staff of a university community in southwest, Nigeria. A cross-sectional survey using an anonymous, self-designed, online KAP questionnaire was conducted from April 18 to May 31, 2020. Purposive and chain referral 
sampling techniques were used to recruit respondents from the teaching and non-teaching categories of the university. The KAP questionnaire consisted of ten knowledge questions regarding the identification of clinical characteristics, transmission and prevention of COVID19. The questions on attitude (15) and perception (10) assessed respondents on adherence to policies and their views on government efforts to contain the spread of the infection respectively. A total of 125 (teaching) and 102 (non-teaching) staff responded. The respondents had scientific (78.8 \%) and non-scientific (28.2\%) work background. Approximately $59.1 \%$ of the respondents were males. The mean knowledge and positive attitude levels were $70.8 \%$ $(\mathrm{SD} \pm 9.6 \%)$ and $83.1 \%(\mathrm{SD} \pm 13.07 \%)$ respectively. Significant differences in the knowledge mean scores were observed for demographic categories such as educational qualification $(\mathrm{p}=$ 0.0006), staff work category $(\mathrm{p}=<0.0001)$, work background $(\mathrm{p}=<0.0001)$, and type of lockdown $(\mathrm{p}=0.0271)$. Most of the respondents $(85.3 \%)$ opined that COVID-19 was a biological weapon and viewed the lockdown as necessary $(81.5 \%)$. However, they thought that the Nigerian government was not doing enough to mitigate COVID-19 spread. The perception of COVID-19 in the university community bear implications across public health initiatives, compliance with precautionary behaviour and bilateral relations with foreign nations.

Keywords: Attitude; COVID-19; Knowledge; Nigeria; Perception; University

\section{Introduction}

The first known case of the novel coronavirus disease (COVID-19), is traced back to December 2019 in Wuhan, Hubei, China (Tang et al., 2020). As at June 12, 2020, the virus has been reported worldwide, with over 7410510 confirmed cases and 418294 deaths (WHO, 2020). Countries worst hit include USA (112 810 deaths), UK (41 279 deaths), Brazil (39 680 deaths), Italy (34 167 deaths), France (29 284 deaths) and Spain (27 136 deaths) (WHO, 2020). The World Health Organisation (WHO) declared the outbreak a public health emergency of 
international concern on January 30, 2020 and recognised it as a pandemic on March 11, 2020 (Sohrabi et al., 2020).

All African countries have recorded COVID-19 infection. South Africa has the highest number of confirmed cases (58 568) with 1284 deaths, followed by Nigeria (14 554 confirmed cases with 387 deaths) (WHO, 2020) as at June 12. The index case of COVID-19 in Nigeria was confirmed by the Nigeria Centre for Disease Control (NCDC) in Lagos State on February 27. In Ogun State, the closest neighbouring state to Lagos, another patient who had been in contact with the index case was identified shortly after. The disease has since spread to 35 of the 36 states of Nigeria and the federal capital territory (FCT).

COVID-19 is caused by the severe acute respiratory syndrome coronavirus 2 (SARS-CoV-2), a novel virus closely related to SARS-CoV, pangolin and bat coronaviruses (Li et al., 2020). The virus is mainly spread during close contact and by respiratory droplets produced when an infected person coughs or sneezes. It is contagious from both symptomatic and asymptomatic carriers. Common symptoms include fever, cough and shortness of breath and the incubation period ranges from two to 14 days (Huang et al., 2020). To date, there is no known vaccine or specific antiviral therapy and management remains largely symptomatic and supportive. This is projected to likely cost the global economy US\$1 trillion this year (Zetzsche, 2020).

Consequent upon this, the WHO advised on several methods to help curb the virus and save health systems globally from complete collapse. Recommended non-pharmaceutical interventions include frequent hand washing, maintaining personal hygiene, social/physical distancing, monitoring and self-isolation of possible contacts (Olapegba et al., 2020). As with several countries globally, the Nigerian President signed the COVID-19 Regulations 2020 declaring the disease as a "dangerous infectious disease" and imposed a nationwide lockdown from March 30, 2020. This involved restrictions on international, national and inter-state 
transport links, market, office or business transactions, educational institutions (primary, secondary and tertiary), sports, religious and all other related social gatherings (Olapegba et al., 2020). Total lockdown (stay-at-home order) was imposed on Lagos, Ogun and FCT for an initial period of two weeks and on April 13, it was extended for another two weeks (Izah et al., 2020). A phased and gradual easing of the lockdown was instituted from May 4.

Considering the grave impact of COVID-19 pandemic on the social, economic and political structure of the world, it has been likened to natural disasters and war outbreaks (Morganstein \& Ursano, 2020; Fiorillo \& Gorwood, 2020). Its emergence and spread, caused confusion, anxiety and fear among the general public (Roy et al., 2020). Furthermore, the recommended ways of containing the spread of the virus such as self-isolation, quarantine, social distancing and the stigma associated with infected persons, put many people at risk of experiencing significant psychological distress (Fiorillo \& Gorwood, 2020). By assessing people's knowledge about diseases, deeper insights into public perception and practices can be gained, thereby helping to identify attributes that influence adopting healthy practices and responsive behaviour (Richards 2017; Al-Hanawi et al., 2020). It is also important in identifying gaps and strengthening ongoing prevention efforts.

To the best of our knowledge, this study represents the first one examining the knowledge, attitude and perception (KAP) as well as concerns about COVID-19 among members of staff of a university community in Nigeria. Universities offer the society an opportunity to reflect on the critical, social, economic, cultural, moral and spiritual issues facing humanity. Its staff members contribute to national development through dissemination of specialized knowledge and skills and guiding the young generation. The findings of this study are expected to provide useful information about KAP to policymakers at this critical time. It may also inform public health officials on further public health interventions, awareness, and policy improvements pertaining to the COVID-19 pandemic. 


\section{Materials and methods}

\subsection{The study area}

The Federal University of Agriculture, Abeokuta (FUNAAB) (Figure 1), a unique and leading institution, is one of the three specialized Universities of Agriculture in Nigeria. It was established on January 1, 1988 with the triple mandate of teaching, research and extension (knowledge and technology transfer).

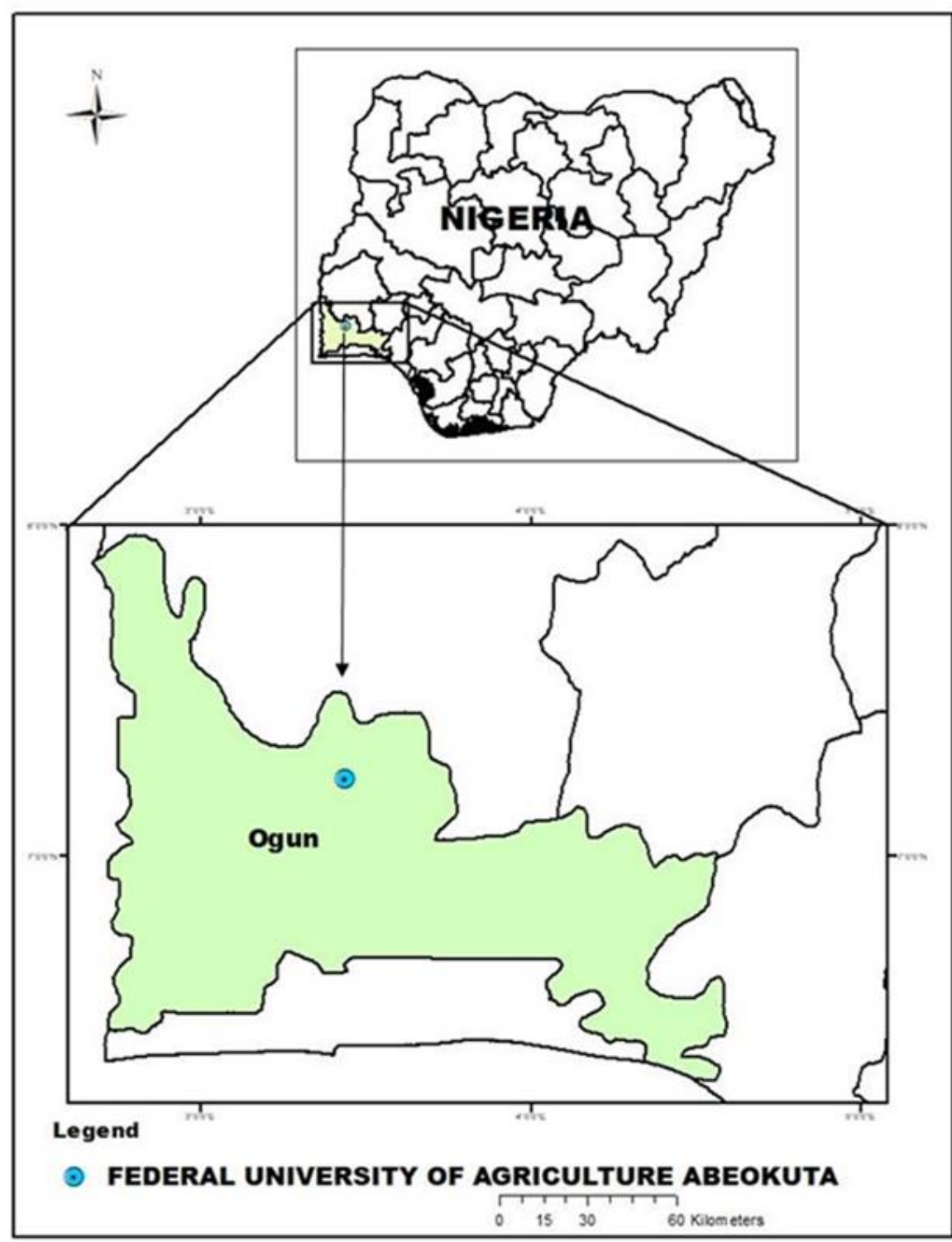

Figure 1: Map of the Federal University of Agriculture, Abeokuta, Ogun State, Nigeria 


\subsection{Ethical approval}

The study was conducted in accordance with internationally accepted principles. Ethical approval was obtained from the College of Veterinary Medicine, FUNAAB Research Ethics Committee (Ref. number: FUNAAB/COLVET/CREC/2020/04/01).

\subsection{The study population}

Members of staff of FUNAAB belonging to any one of the four associations in the University. 1) Academic Staff Union of Universities (ASUU) comprising the teaching staff, 2) National Association of Academic Technologists (NAAT), 3) Senior Staff Association of Nigerian Universities (SSANU) and 4) Non-Academic Staff Union of Education and Associated Institutions (NASU).

\subsection{Study design and sampling}

A cross-sectional survey using a validated, semi-structured, online questionnaire was used for data collection (Geldsetzer, 2020). Purposive and chain referral sampling techniques were employed to recruit respondents.

As the survey was done during the lockdown, it was very difficult to physically access people due to the restriction of movement. As such, a questionnaire using Google online survey platform was designed by the investigators. A detailed informed consent was sought at the beginning of the online questionnaire and consent was a prerequisite to continue in the survey. The link of the questionnaire was sent via social media (WhatsApp and Telegram posts) to the different chairmen of the associations, deans and directors of colleges, directorates and units in FUNAAB. The prospective respondents were then encouraged to roll out the survey to as many colleagues as possible. Thus, the link was forwarded to people apart from the first point of contact. 
The online survey ran from April 18 (second phase of lockdown) to May 31 (last week of the lockdown in Ogun State). Participation was voluntary and anonymous, with respondents being assured that their responses would remain confidential and used only for research purposes. Two hundred and twenty - seven (227) correctly filled questionnaire was submitted through the Google form, exported unto excel spreadsheet and processed for statistical analysis.

\subsection{Questionnaire and data collection}

The questionnaire contained four sections. The first section consisted of information assessing socio-demographic attributes of the respondents on variables such as age, sex, staff work category, marital status, educational qualification, religion, number of persons and pets in household and state of residence during the lockdown.

The second section assessed the general knowledge about COVID-19. It consisted of ten questions regarding the source of COVID-19, modes of transmission, symptoms, susceptible individuals and methods of prevention, control and treatment.

In section three, the evaluation of respondents' attitude was performed by 15 questions. Parameters evaluated if the non-pharmaceutical interventions and social distancing rules were adhered to, if updates on COVID-19 were sought and how activities were undertaken during the lockdown and prophylactic use of drugs (orthodox or herbal). Using a Likert scale of 1-5 (1-lowest negative effect, 5- highest negative effect), the effect of the lockdown on the mental, physical, social, financial well-being and sexual performances of the respondents were also assessed.

The final section evaluated which of the impacts of COVID-19 individuals were most concerned about- the source, the spread, the actions or inactions of the Nigerian government and/or international bodies. The rate of satisfaction to the COVID-19 relief package of the 
government and other current measures taken by the Nigerian government during the lockdown was also assessed.

\subsection{Data analysis}

Descriptive statistics were calculated for all variables in the forms of frequencies and proportions/percentages using Microsoft Excel ${ }^{\mathrm{R}}$ (2013). Respondents' knowledge and attitude towards COVID-19 was arrived at by summing correct responses, generating a maximum possible score of 27 and 11 respectively. A correct response to an item was assigned 1 point, while an incorrect/not sure response was assigned 0 point. Scores were then converted to percentages and a cut off $<70 \%$ and $\geq 70 \%$ was used to categorise respondents as having poor and good levels respectively. Normality tests were conducted on continuous variables using Kolmogorov-Smirnov (> 0.05) and Shapiro-Wilk (> 0.05) and data expressed as mean and standard deviation (Mean \pm SD). Independent samples t-tests and one-way analysis of variance (ANOVA) were utilised to determine the differences in knowledge and attitude scores between groups of demographic variables.

All variables were recoded into binary outcomes for further analysis. Associations between the socio-demographics of respondents and knowledge level, knowledge level with social, financial, physical, and mental impact of the pandemic were determined using chi-square tests and binary logistic regression analysis (BLRA) (SPSS® 21 analysis software package IBM, Inc.). A p $<0.05$ was considered statistically significant and crude odds ratios were computed to determine the strength of associations between variables at $95 \%$ confidence intervals (CIs). All illustrations were performed with Graphpad prism 8.0. 


\section{Results}

\subsection{Respondents' socio-demographics}

Responses were received from 227 respondents comprising $125(55.1 \%)$ teaching staff and $59.9 \%$ males. Approximately $40.1 \%$ of the respondents were within 40 - 49 years and $88.1 \%$ of them were married. All respondents (100\%) had completed the primary school leaving certificate programme. A little over half of the respondents had household members between 5 and $10(52.0 \%)$. Owning household pets was observed to be less common (23.3\%). Most of the respondents resided within Ogun State $(81.5 \%)$, and a total or partial lockdown was instituted in many of the respondents' (83.3\%) states of residence (Table 1). 


\section{Table 1}

Sociodemographic data of members of staff of the Federal University of Agriculture, Abeokuta, Ogun State, Nigeria

\begin{tabular}{|c|c|c|c|}
\hline $\mathrm{S} / \mathrm{N}$ & $\begin{array}{l}\text { Variables } \\
(\mathrm{n}=227)\end{array}$ & Proportions (\%) & $95 \% \mathrm{CI}$ \\
\hline 1 & $\begin{array}{l}20-29 \\
30-39 \\
40-49 \\
50-59 \\
60-69 \\
>70\end{array}$ & $\begin{array}{l}9(4.0) \\
58(25.6) \\
91(40.1) \\
61(26.9) \\
7(3.1) \\
1(0.4)\end{array}$ & $\begin{array}{l}2.0-7.7 \\
20.3-31.6 \\
33.9-46.6 \\
21.5-33.0 \\
1.4-6.3 \\
<0.01-2.7\end{array}$ \\
\hline 2 & $\begin{array}{l}\text { Female } \\
\text { Male }\end{array}$ & $\begin{array}{r}91(40.1) \\
136(59.9)\end{array}$ & $\begin{array}{l}33.9-46.6 \\
53.4-66.1\end{array}$ \\
\hline 3 & $\begin{array}{l}\text { Educational qualification } \\
\text { Primary } \\
\text { Secondary } \\
\text { Bachelor's } \\
\text { Master's } \\
\text { PhD } \\
\text { Others }\end{array}$ & $\begin{array}{l}0(0.0) \\
5(2.2) \\
47(20.7) \\
62(27.3) \\
89(39.2) \\
24(10.6)\end{array}$ & $\begin{array}{l}0 \\
0.8-5.2 \\
15.9-26.5 \\
21.9-33.4 \\
33.0-45.7 \\
7.16-15.3\end{array}$ \\
\hline 4 & $\begin{array}{l}\text { Staff work categories } \\
\text { Academic (Teaching) } \\
\text { Non-Teaching }\end{array}$ & $\begin{array}{l}125(55.1) \\
102(44.9)\end{array}$ & $\begin{array}{l}48.6-61.4 \\
38.6-51.4\end{array}$ \\
\hline 5 & $\begin{array}{l}\text { Work background } \\
\text { Scientific } \\
\text { Non-Scientific }\end{array}$ & $\begin{array}{r}163(71.8) \\
64(28.2) \\
\end{array}$ & $\begin{array}{l}65.6-77.3 \\
22.7-34.4\end{array}$ \\
\hline 6 & $\begin{array}{l}\text { Marital status } \\
\text { Married } \\
\text { Single } \\
\text { Divorced } \\
\text { Widowed } \\
\end{array}$ & $\begin{array}{c}200(88.1) \\
24(10.6) \\
0(0.0) \\
3(1.3)\end{array}$ & $\begin{array}{l}83.2-91.7 \\
7.2-15.3 \\
0.3 \text { to } 4.0\end{array}$ \\
\hline 7 & $\begin{array}{ll}\text { Religion } & \\
& \text { Christianity } \\
& \text { Islam } \\
& \text { Traditional } \\
& \text { Others }\end{array}$ & $\begin{array}{l}194(85.5) \\
32(14.1) \\
1(0.4) \\
0(0.0)\end{array}$ & $\begin{array}{l}80.3-89.5 \\
10.1-19.3 \\
<0.01-2.7 \\
0\end{array}$ \\
\hline 8 & $\begin{array}{l}\text { Number of household members } \\
\text { Less than } 5 \\
5-10 \\
10 \text { and above }\end{array}$ & $\begin{array}{l}107(47.1) \\
118(52.0) \\
2(0.9)\end{array}$ & $\begin{array}{l}40.7-53.6 \\
45.5-58.4 \\
0.03-3.4\end{array}$ \\
\hline 9 & $\begin{array}{l}\text { Do you have any household pets? } \\
\text { Yes } \\
\text { No }\end{array}$ & $\begin{array}{l}53(23.3) \\
174(76.7)\end{array}$ & $\begin{array}{l}18.3-29.3 \\
70.7-81.7\end{array}$ \\
\hline 10 & $\begin{array}{l}\text { State of residence during the lockdown } \\
\text { Ogun } \\
\text { Oyo } \\
\text { Ekiti } \\
\text { Osun } \\
\text { Lagos } \\
\text { Kogi } \\
\text { Kwara } \\
\text { Abia } \\
\text { Enugu } \\
\text { Kaduna } \\
\text { Others }\end{array}$ & $\begin{array}{l}185(81.5) \\
20(8.8) \\
1(0.4) \\
3(1.3) \\
7(3.1) \\
1(0.4) \\
1(0.4) \\
2(0.9) \\
3(1.3) \\
2(0.9) \\
2(0.9)\end{array}$ & $\begin{array}{l}76.7-86.7 \\
5.8-13.4 \\
<0.01-2.7 \\
0.3-4.0 \\
1.4-6.4 \\
<0.01-2.7 \\
<0.01-2.7 \\
0.03-3.4 \\
0.3-4.0 \\
0.03-3.4 \\
0.03-3.4\end{array}$ \\
\hline 11 & $\begin{array}{l}\text { Type of lockdown where resident } \\
\text { Partial } \\
\text { Total }\end{array}$ & $\begin{array}{l}189(83.3) \\
38(16.7)\end{array}$ & $\begin{array}{l}77.8-87.6 \\
12.41-22.2\end{array}$ \\
\hline
\end{tabular}




\subsection{Knowledge assessment of COVID-19}

Table 2 showed that the common sources of information on COVID-19 were TV/radio (85.5 $\%)$ and social media (79.3\%). Many of the respondents could correctly identify COVID-19 as viral (97.4\%), emerging (86.8\%) and infectious (93.7\%). Most common symptoms accurately identified by respondents was dry cough (93.7\%), shortness of breath $(92.1 \%)$, fever $(87.7 \%)$, and sore throat $(78.9 \%)$.

The mean knowledge score for participants was $19.1 \pm 2.6(70.8 \pm 9.6 \%$, range $33.3-92.6 \%)$. About $59.5 \%$ of respondents scored above the $\geq 70 \%$ cut off for general good knowledge, which was averagely acceptable. The t-tests and ANOVA, as well as Chi-square analysis showed that knowledge score was not statistically significant across gender $(\mathrm{p}=0.1833)$, whereas, age groups $(p=0.04)$, educational qualification $(p=0.0006 ; p=0.01)$, staff work category $(\mathrm{p}=<0.0001 ; \mathrm{p}=0.01)$, work background $(\mathrm{p}=<0.0001)$, staff residence $(\mathrm{p}=0.005)$ and type of lockdown $(\mathrm{p}=0.0271 ; \mathrm{p}=0.048)$ were statistically significant respectively. Dunnett's multiple comparisons test showed a significantly higher knowledge score for respondents holding Master's $(\mathrm{p}=0.0163)$ and $\mathrm{PhD}$ degrees $(\mathrm{p}=0.0100)($ Table 3,4$)$.

For BLRA, respondents within the age group $<40-49$ years were about 2.2 times $(\mathrm{OR}=2.18$, 95\%CI: $1.09-4.38 ; \mathrm{p}=0.03$ ) more likely to have good knowledge on COVID-19 than older ones. Also, respondents resident within Ogun State were about 2.5 times $(\mathrm{OR}=2.43,95 \% \mathrm{CI}$ : 1.04-5.67, $\mathrm{p}=0.04$ ) more likely to have good knowledge of COVID-19. Although marginally significant, respondents having household pets have about 1.91 higher odds of good knowledge of COVID-19 (OR=1.91, 95\%CI: $0.98-3.76, \mathrm{p}=0.06)$ (Table 4). 


\section{Table 2}

Different sources of information and respondents' knowledge responses to COVID-19

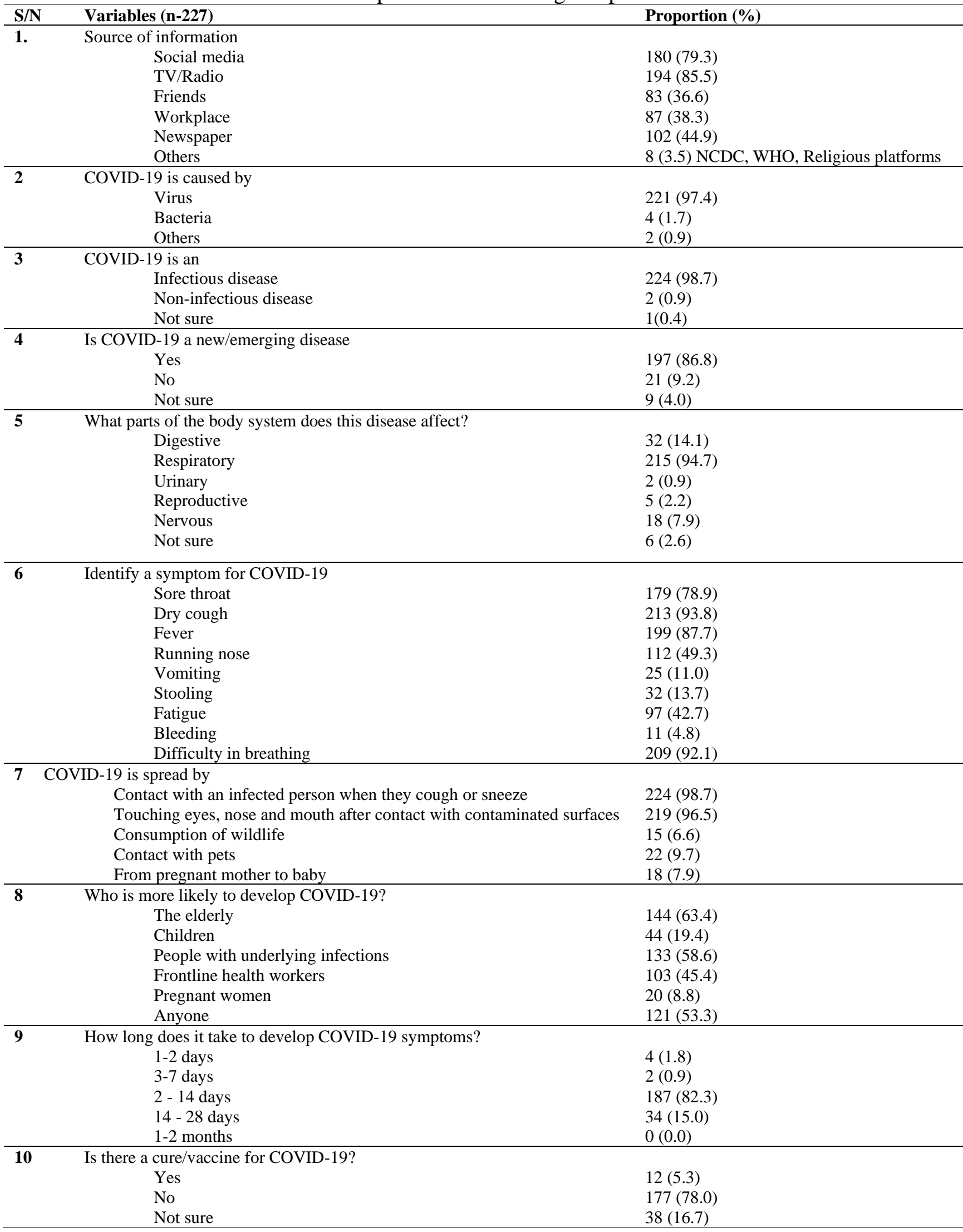

Key: NCDC - Nigeria Centre for Disease Control; WHO - World Health Organisation; Respondents- Members of staff of the Federal University of Agriculture, Abeokuta, Ogun State, Nigeria 


\section{Table 3}

Demographic characteristics of respondents and their knowledge and attitude score

\begin{tabular}{|c|c|c|c|c|c|c|}
\hline $\mathbf{S} / \mathbf{N}$ & Variables $(\mathrm{n}=\mathbf{2 2 7})$ & $\begin{array}{l}\text { Proportions } \\
(\%)\end{array}$ & $\begin{array}{l}\text { Knowledge } \\
\text { level }(\%) \\
\text { (Mean } \\
\pm \text { SD) }\end{array}$ & $\begin{array}{l}\text { P value } \\
\text { (ANOVA } \\
\text { Independent } \\
\text { T-test) }\end{array}$ & $\begin{array}{l}\text { Attitude level } \\
(\%) \\
(\text { Mean } \pm \text { SD) }\end{array}$ & $\begin{array}{l}\text { P value } \\
\text { (ANOVA } \\
\text { Independent T- } \\
\text { test) }\end{array}$ \\
\hline 1 & $\begin{array}{l}20-29 \\
30-39 \\
40-49 \\
50-59 \\
60-69 \\
\geq 70\end{array}$ & $\begin{array}{l}9(4.0) \\
58(25.6) \\
91(40.1) \\
61(26.9) \\
7(3.1) \\
1(0.4)\end{array}$ & $\begin{array}{l}65.4 \pm 8.3 \\
71.0 \pm 10.4 \\
71.1 \pm 9.6 \\
70.3 \pm 8.6 \\
70.9 \pm 7.8 \\
74.1 \pm 0.0\end{array}$ & 0.5362 & $\begin{array}{l}74.7 \pm 23.1 \\
84.0 \pm 12.4 \\
81.9 \pm 12.5 \\
84.1 \pm 12.9 \\
89.6 \pm 6.3 \\
90.9 \pm 0.0\end{array}$ & 0.2124 \\
\hline 2 & $\begin{array}{l}\text { Female } \\
\text { Male }\end{array}$ & $\begin{array}{r}91(40.1) \\
136(59.9)\end{array}$ & $\begin{array}{l}71.7 \pm 9.1 \\
70.0 \pm 9.6\end{array}$ & $<0.1833$ & $\begin{array}{l}83.9 \pm 13.2 \\
82.5 \pm 13.0\end{array}$ & 0.4209 \\
\hline 3 & $\begin{array}{l}\text { Educational } \\
\text { qualification } \\
\text { Secondary } \\
\text { Bachelor's } \\
\text { Master's } \\
\text { PhD } \\
\text { Others } \\
\end{array}$ & $\begin{array}{l}5(2.2) \\
47(20.7) \\
62(27.3) \\
89(39.2) \\
24(10.6)\end{array}$ & $\begin{array}{l}60.7 \pm 5.0 \\
67.5 \pm 9.6 \\
72.1 \pm 9.0 \\
72.7 \pm 9.2 \\
67.6 \pm 8.7 \\
\end{array}$ & $0.0006^{*}$ & $\begin{array}{l}83.6 \pm 13.5 \\
82.0 \pm 14.3 \\
82.2 \pm 13.1 \\
83.9 \pm 12.3 \\
84.1 \pm 14.0 \\
\end{array}$ & 0.9071 \\
\hline 4 & $\begin{array}{l}\text { Staff work } \\
\text { categories } \\
\text { Academic } \\
\text { (Teaching) } \\
\text { Non-Teaching }\end{array}$ & $\begin{array}{l}125(55.1) \\
102(44.9)\end{array}$ & $\begin{array}{l}73.0 \pm 9.4 \\
67.8 \pm 8.7\end{array}$ & $<0.0001^{*}$ & $\begin{array}{l}82.4 \pm 13.6 \\
83.9 \pm 12.5\end{array}$ & 0.4010 \\
\hline 5 & $\begin{array}{c}\text { Work background } \\
\text { Scientific } \\
\text { Non- } \\
\text { Scientific } \\
\end{array}$ & $\begin{array}{r}163(71.8) \\
64(28.2)\end{array}$ & $\begin{array}{l}71.8 \pm 9.1 \\
67.8 \pm 9.7\end{array}$ & $<0.0001^{*}$ & $\begin{array}{l}82.8 \pm 12.6 \\
83.6 \pm 14.2\end{array}$ & 0.6617 \\
\hline 6 & $\begin{array}{c}\text { Marital status } \\
\text { Married } \\
\text { Single } \\
\text { Widowed }\end{array}$ & $\begin{array}{r}200(88.1) \\
24(10.6) \\
3(1.3)\end{array}$ & $\begin{array}{l}70.6 \pm 9.3 \\
70.8 \pm 10.7 \\
75.3 \pm 5.7\end{array}$ & 0.6916 & $\begin{array}{l}82.9 \pm 12.6 \\
83.0 \pm 17.2 \\
93.9 \pm 5.3\end{array}$ & 0.3497 \\
\hline 7 & $\begin{array}{l}\text { Religion }(\mathrm{n}=226) \\
\text { Christianity } \\
\text { Islam }\end{array}$ & $\begin{array}{l}194(85.5) \\
32(14.1)\end{array}$ & $\begin{array}{l}70.4 \pm 9.5 \\
71.9 \pm 9.4\end{array}$ & 0.4081 & - & 0.2545 \\
\hline 8 & $\begin{array}{l}\text { Number of } \\
\text { household members } \\
\text { Less than } 5 \\
5-10 \\
10 \text { and above } \\
\end{array}$ & $\begin{array}{l}107(47.1) \\
118(52.0) \\
2(0.9) \\
\end{array}$ & $\begin{array}{l}71.8 \pm 10.2 \\
72.2 \pm 7.9 \\
69.6 \pm 8.7 \\
\end{array}$ & 0.2141 & $\begin{array}{l}82.1 \pm 14.2 \\
83.7 \pm 11.9 \\
100.0 \pm 0.0 \\
\end{array}$ & 0.1205 \\
\hline 9 & $\begin{array}{c}\text { Do you have any } \\
\text { household pets? } \\
\text { Yes } \\
\text { No }\end{array}$ & $\begin{array}{l}53(23.3) \\
174(76.7)\end{array}$ & $\begin{array}{l}69.5 \pm 11.1 \\
71.0 \pm 8.9\end{array}$ & 0.3130 & $\begin{array}{l}81.1 \pm 14.1 \\
83.6 \pm 12.7\end{array}$ & 0.2211 \\
\hline 10 & $\begin{array}{l}\text { Type of lockdown } \\
\text { where resident } \\
\text { Partial } \\
\text { Total }\end{array}$ & $\begin{array}{l}189(83.3) \\
38(16.7)\end{array}$ & $\begin{array}{l}71.3 \pm 9.2 \\
67.6 \pm 10.1\end{array}$ & $0.0271^{*}$ & $\begin{array}{l}83.0 \pm 13.2 \\
83.5 \pm 12.7\end{array}$ & 0.8232 \\
\hline
\end{tabular}

Key: Respondents - Members of staff of the Federal University of Agriculture, Abeokuta, Ogun State, Nigeria 


\section{Table 4}

Association between respondents' demographic characteristics and knowledge about COVID19

\begin{tabular}{|c|c|c|c|c|}
\hline Variable & Category & OR & $95 \% \mathrm{CI}$ & P value \\
\hline Age groups in years & $\begin{array}{l}<40-49 \\
>49\end{array}$ & $\begin{array}{l}2.18 \\
1\end{array}$ & $1.09-4.38$ & $0.03 *$ \\
\hline Staff work categories & $\begin{array}{l}\text { Academic (Teaching) } \\
\text { Non-Teaching }\end{array}$ & $\begin{array}{l}0.59 \\
1\end{array}$ & $0.29-1.17$ & 0.13 \\
\hline Work background & $\begin{array}{l}\text { Scientific } \\
\text { Non-Scientific }\end{array}$ & $\begin{array}{l}0.93 \\
1\end{array}$ & $0.43-2.01$ & 0.86 \\
\hline Number of household members & $\begin{array}{l}<5 \\
\geq 5\end{array}$ & 0.73 & $0.40-1.34$ & 0.31 \\
\hline Household pets & $\begin{array}{l}\text { Yes } \\
\text { No }\end{array}$ & $\begin{array}{l}1.91 \\
1\end{array}$ & $0.98-3.76$ & 0.06 \\
\hline $\begin{array}{l}\text { State of residence during the } \\
\text { lockdown }\end{array}$ & $\begin{array}{l}\text { Within Ogun } \\
\text { Outside Ogun }\end{array}$ & $\begin{array}{l}2.43 \\
1\end{array}$ & $1.04-5.67$ & $0.04 *$ \\
\hline Lockdown type & $\begin{array}{l}\text { Partial } \\
\text { Total }\end{array}$ & $\begin{array}{l}0.51 \\
1\end{array}$ & $0.237-\quad 1.097$ & 0.09 \\
\hline
\end{tabular}

Key: OR - Odd Ratio; CI - Confidence Interval; ${ }^{\mathrm{P}} \leq 0.05$; Reference $=1.00$; Respondents - Members of staff of the Federal University of Agriculture, Abeokuta, Ogun State, Nigeria 


\subsection{Attitude during the lockdown}

Respondents were asked 15 questions to assess their compliance with the non-pharmaceutical interventions and the impact on their social, financial, physical and mental health. Most of the respondents complied with the use of nose/face masks, stay at home policy, social distancing and regular hand wash (Figure 2). A total of 192 (84.6\%) and 203 respondents (89.4\%) used hand sanitizers, and soap and water respectively. Majority of the respondents rarely left their houses during the lockdown $(84.6 \%)$. When they did, they mostly went to the market $(71.8 \%)$ and the less visited places were hospitals/clinics (10.6\%) and religious houses (5.3\%). Other preventive measures taken to prevent infection were eating healthy foods, use of hand gloves, prayers and use of dihydroxy chloroquine. To boost their immunity, about $7.5 \%$ of the respondents consumed multivitamins and $2.2 \%$ took herbal mixtures.

Respondents kept themselves busy during the lockdown by spending time with family (71.4 $\%)$, watching movies (70.9\%), reading (70.5\%), engaging in social media activities $(69.2 \%)$, working out (58.6\%), working from home (47.3\%), playing with their pets $(2.2 \%)$ and visiting friends.

The mean attitude score was $9.1 \pm 1.4(83.1 \% \pm 13.07$, range $36.4-100.0 \%)$. Most of the respondents had good attitude levels $(88.0 \%)$. Attitude scores among different demographic characteristics were statistically non-significant, showing respondents had a similar level of attitude across board (Table 3).

The social (33.5 \%), physical (38.8 \%) and mental impact (29.5\%) were self - reported as being moderately affected by the lockdown. Contrastingly, respondents' $(31.3 \%)$ financial/cost of living suffered a much higher impact (Figure 3). The teaching staff are 0.43 less likely (OR= 0.43, 95\%CI: $0.19-0.98, p=0.045)$ to have a low social impact due to COVID-19. Also, respondents with household pets were 0.49 less likely $(\mathrm{OR}=0.49,95 \% \mathrm{CI}: 0.24-1.00, \mathrm{p}=$ 
0.049) to have a low social impact. Although only marginally significant, respondents under partial lockdown had 2.13 increased odds $(\mathrm{OR}=2.13,95 \% \mathrm{CI}: 0.95-4.79, \mathrm{p}=0.07)$ for low social impact than those under total lockdown (Table 5).

Respondents with family household members less than five were about three times more likely $(\mathrm{OR}=3.14,95 \% \mathrm{CI}: 1.33-7.38, \mathrm{p}=0.009)$ to have a low financial impact due to COVID-19 lockdown than those with more than five members. Also, the non-teaching staff of the university were about 2.56 more likely $(\mathrm{OR}=0.39,95 \% \mathrm{CI}: 0.14-1.08, \mathrm{p}=0.07)$, to experience a low financial impact, but marginally significant (Table 5).

Staff members with scientific background were half less likely (OR=0.50, 95\%CI: 0.24 -1.05, $\mathrm{p}=0.07)$ to have low mental health impact due to the pandemic than those with no scientific background. Household with members less than five were about 1.74 more likely $(\mathrm{OR}=1.74$, 95\%CI: $0.98-3.08, \mathrm{p}=0.06)$ to have a low mental impact than those with more than or equal to five members (Table 5).

Frequency of sexual performance was observed to be the same as before the pandemic in 46.7 $\%$ of the respondents, $9.7 \%$ reported it was at its lowest ever and $12.8 \%$ at its highest frequency. The respondents reported they majorly suffered anxiety/worry/fear/restlessness $(59.9 \%)$ and boredom (48.0\%). More of the respondents were optimistic while only $4.8 \%$ were angry or pessimistic $(1.8 \%)$ about the pandemic. 


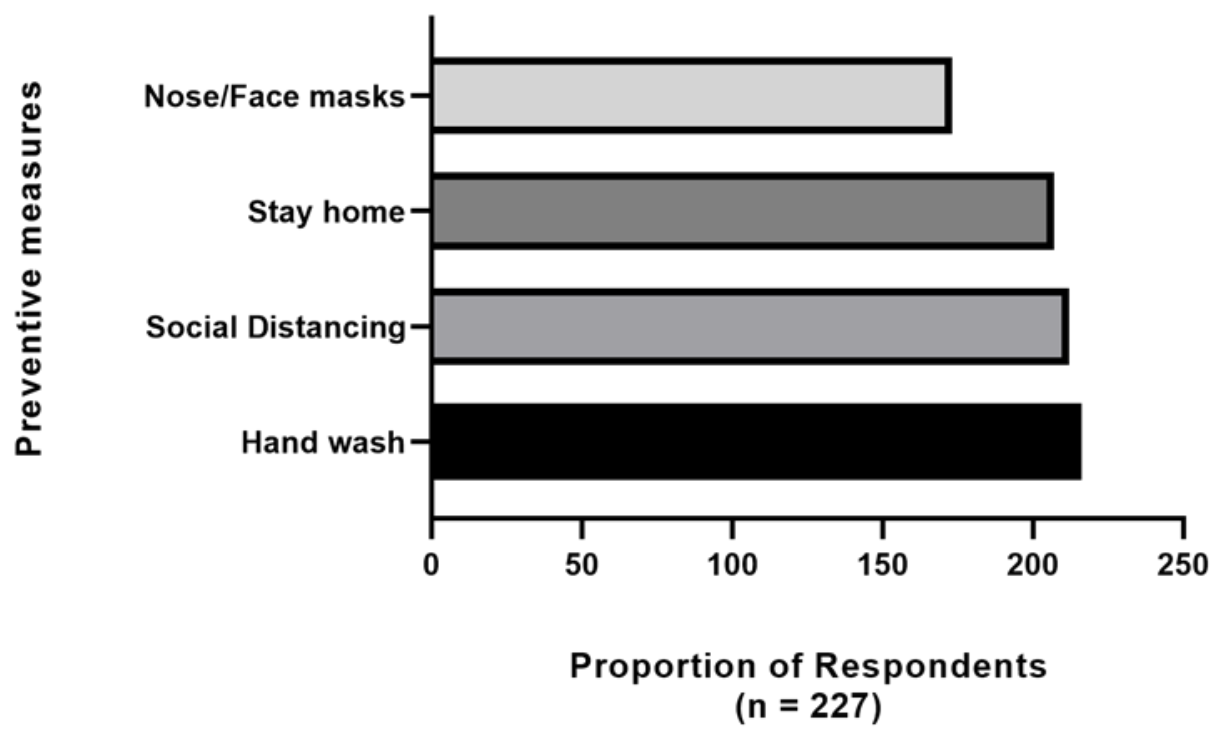

Figure 2. Preventive measures complied with by respondents

Key: Respondents - Members of staff of the Federal University of Agriculture, Abeokuta, Ogun State, Nigeria 


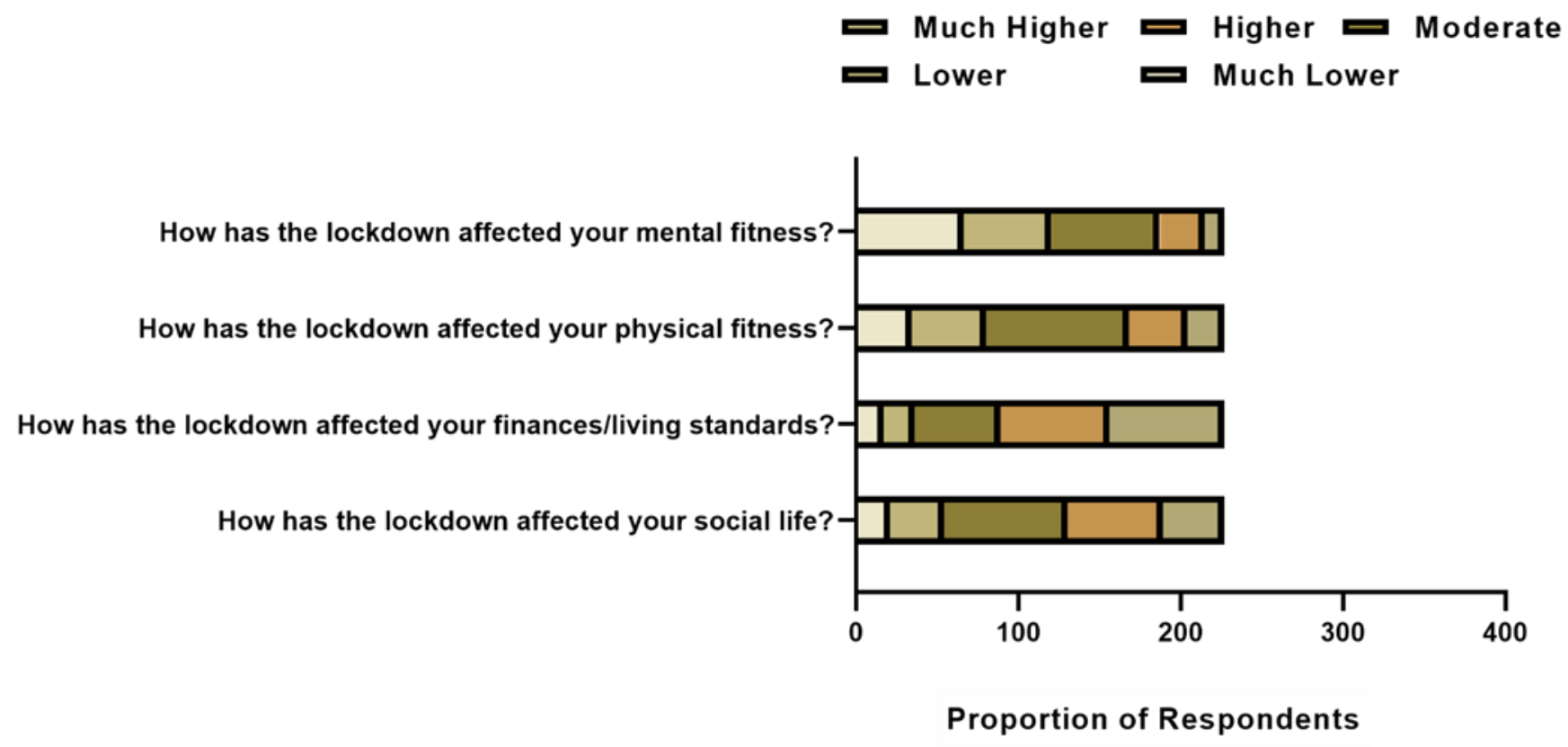

Figure 3. The effect of lockdown on respondents' social, financial, physical and mental wellbeing

Key: Respondents - Members of staff of the Federal University of Agriculture, Abeokuta, Ogun State, Nigeria 


\section{Table 5}

Association between selected respondents' demographic profiles and social, financial and mental impact due to COVID-19 pandemic

\begin{tabular}{|c|c|c|c|c|c|c|c|c|c|c|}
\hline \multirow[t]{2}{*}{ Variable } & \multirow[t]{2}{*}{ Category } & \multicolumn{3}{|c|}{ Social impact } & \multicolumn{3}{|c|}{ Financial impact } & \multicolumn{3}{|c|}{ Mental impact } \\
\hline & & OR & $95 \% \mathrm{CI}$ & $P$ value & OR & $95 \% \mathrm{CI}$ & P value & OR & $95 \% \mathrm{CI}$ & P value \\
\hline Age groups in years & $\begin{array}{l}<40-49 \\
>49\end{array}$ & $\begin{array}{l}1.35 \\
1\end{array}$ & $0.60-3.02$ & 0.47 & $\begin{array}{l}0.84 \\
1\end{array}$ & $0.34-2.12$ & 0.72 & $\begin{array}{l}1.68 \\
1\end{array}$ & $0.87-3.26$ & 0.12 \\
\hline Sex & $\begin{array}{l}\text { Female } \\
\text { Male }\end{array}$ & $\begin{array}{l}0.78 \\
1\end{array}$ & $0.41-1.49$ & 0.45 & $\begin{array}{l}0.99 \\
1\end{array}$ & $0.45-2.19$ & 0.98 & $\begin{array}{l}0.75 \\
1\end{array}$ & $0.43-1.33$ & 0.33 \\
\hline $\begin{array}{l}\text { Educational } \\
\text { qualification }\end{array}$ & $\begin{array}{l}\text { Secondary } \\
\text { Post-Secondary }\end{array}$ & $\begin{array}{l}0.77 \\
1 \\
\end{array}$ & $0.08-7.73$ & 0.83 & - & - & - & $\begin{array}{l}1.24 \\
1 \\
\end{array}$ & $0.18-8.69$ & 0.83 \\
\hline $\begin{array}{l}\text { Staff work } \\
\text { categories }\end{array}$ & $\begin{array}{l}\text { Teaching } \\
\text { Non-Teaching }\end{array}$ & $\begin{array}{l}0.43 \\
1\end{array}$ & $0.19-0.98$ & $0.045^{*}$ & $\begin{array}{l}0.39 \\
1\end{array}$ & $0.14-1.08$ & 0.07 & $\begin{array}{l}1.03 \\
1\end{array}$ & $0.52-2.04$ & 0.93 \\
\hline Work background & $\begin{array}{l}\text { Scientific } \\
\text { Non-Scientific }\end{array}$ & $\begin{array}{l}1.00 \\
1\end{array}$ & $0.40-2.51$ & 0.99 & $\begin{array}{l}0.62 \\
1\end{array}$ & $0.17-2.27$ & 0.47 & $\begin{array}{l}0.50 \\
1\end{array}$ & $0.24-1.05$ & 0.07 \\
\hline Marital status & $\begin{array}{l}\text { Married } \\
\text { Not Married } \\
\end{array}$ & $\begin{array}{l}1.40 \\
1 \\
\end{array}$ & $0.48-4.10$ & 0.54 & $\begin{array}{l}1.81 \\
1 \\
\end{array}$ & $0.58-5.62$ & 0.31 & $\begin{array}{l}0.59 \\
1 \\
\end{array}$ & $0.23-1.49$ & 0.26 \\
\hline $\begin{array}{l}\text { Number of } \\
\text { household members }\end{array}$ & $\begin{array}{l}<5 \\
\geq 5\end{array}$ & $\begin{array}{l}1.46 \\
1\end{array}$ & $0.40-1.34$ & 0.27 & $\begin{array}{l}3.14 \\
1\end{array}$ & $1.33-7.38$ & $0.009^{*}$ & $\begin{array}{l}1.74 \\
1\end{array}$ & $0.98-3.08$ & 0.06 \\
\hline Household pets & $\begin{array}{l}\text { Yes } \\
\text { No }\end{array}$ & $\begin{array}{l}0.49 \\
1\end{array}$ & $0.24-1.00$ & $0.049 *$ & $\begin{array}{l}1.39 \\
1\end{array}$ & $0.53-3.60$ & 0.50 & $\begin{array}{l}0.65 \\
1\end{array}$ & $0.34-1.27$ & 0.21 \\
\hline $\begin{array}{l}\text { State of residence } \\
\text { during the lockdown }\end{array}$ & $\begin{array}{l}\text { Within Ogun } \\
\text { Outside Ogun }\end{array}$ & $\begin{array}{l}0.60 \\
1\end{array}$ & $0.24-1.49$ & 0.27 & $\begin{array}{l}2.03 \\
1\end{array}$ & $0.83-5.00$ & 0.12 & $\begin{array}{l}1.27 \\
1\end{array}$ & $0.61-2.63$ & 0.53 \\
\hline Lockdown type & $\begin{array}{l}\text { Partial } \\
\text { Total }\end{array}$ & $\begin{array}{l}2.13 \\
1\end{array}$ & $0.95-4.79$ & 0.07 & $\begin{array}{l}1.25 \\
1\end{array}$ & $0.46-3.39$ & 0.66 & $\begin{array}{l}1.13 \\
1\end{array}$ & $0.54-2.36$ & 0.74 \\
\hline
\end{tabular}

Key: OR - Odd Ratio; CI - Confidence Interval; ${ }^{*} \mathrm{P} \leq 0.05$; Reference $=1.00$; Respondents - Members of staff of the Federal University of Agriculture, Abeokuta, Ogun State, Nigeria 


\subsection{Perception and concerns about COVID-19}

Approximately 162/227 (85.3\%) of the respondents perceived COVID-19 was likely a laboratory-made or biological weapon. About $19.8 \%$ and $8.8 \%$ slightly believed or were not sure respectively. The global and national situation of the pandemic were of worry to the respondents ( $84.1 \%$ and $53.7 \%$ respectively). The other concerns were increased crime rates (90.7\%), the impact on the economy (88.5\%) and children education (72.2\%), idle children (62.6\%), fear of themselves and family contracting the disease (52.4\%), and domestic violence $(40.1 \%)$.

The majority viewed the lockdown instituted by the government as necessary $(81.5 \%)$, while 71.3\% were not satisfied with the current COVID-19 relief package provided by the government and believed the government was not doing enough in containing the spread of the infection. About $90.5 \%$ of the respondents indicated their readiness to get involved in sharing or contributing their quota if the need arises. 


\section{Discussion}

Nigeria, the most populous country in Africa, has adequately contained many outbreaks of emerging and re-emerging diseases such as poliomyelitis, avian influenza, African swine fever, Yellow fever, Lassa fever and Ebola virus disease (Isere et al., 2015). Nigerians are distinct people with a rich culture of socializing and daily income earning. Movement restriction, physical and social distancing, closure of schools and religious centres and the mandatory use of nose masks associated with COVID-19 greatly impacts the population (Olapegba et al., 2020).

This study assessed the KAP of an ivory tower-based population in Nigeria about COVID-19 pandemic and associated effects. A large proportion of the respondents were aware and knowledgeable about the presence, symptoms and mode of transmission of COVID-19 in Nigeria. This may be due to the educational status of the respondents as over $87 \%$ of them had a bachelor's degree or above. Many of them $(71.8 \%)$ were in scientific work category. In addition, most of the respondents were married and fell within the active middle age group (4049 years). All these stimulate interest and search for knowledge about COVID-19 and its transmission from various sources, to protect themselves and their families. These findings agree with previous studies which reported positive association between knowledge, educational background and age during epidemics/pandemics (Brug et al., 2004; Choi and Yang, 2010, Hussain et al., 2012; Olapegba et al., 2020, Olaseni et al., 2020; Al-Hanawi et al., 2020).

It was also noticed that respondents having household pets had better knowledge of COVID19. This may be due to information obtained from their veterinarians and they are likely to be more cautious. Though COVID-19 infection has been reported in dogs, there are no evidences yet that dogs and cats, can be sources of human infection (Goumenou et al., 2020). 
Our study also identified the mass media (television, radio) and social media as the major sources of information about COVID-19. This indicates that a significant proportion of respondents are largely influenced by media information. Many of the respondents $(81.5 \%)$ live in Ogun State where a total lockdown was instituted, hence, they accessed mass media more frequently. It is important to note that the Nigerian Ministry of Health presently conducts intensive awareness campaign on COVID-19 via the television and various social media in English, French and the three main Nigerian languages (Hausa, Igbo and Yoruba) (NCDC, 2020). The importance of the media in providing vital information during outbreaks has been confirmed by several researchers (Lau et al., 2003; Vartti et al., 2009; Voeten et al., 2009).

According to the Economic Commission for Africa, approximately 300000 to 3.3 million COVID-19 related deaths are predicted to occur in Africa (Berhan, 2020). Fortunately, the mortality rate as at June 12, 2020 is quite low when compared with USA and European countries (Otitoloju et al., 2020). Many factors such as environmental (sunlight, humidity, prevalence of malaria parasite) and genetic have been attributed to it though none has yet been confirmed (Otitoloju et al., 2020). This may have contributed to the general positive and optimistic attitude toward COVID-19.

Respondents with less than five household members were more likely to have a low social, financial and mental impact due to COVID-19. This is not surprising as lesser amount will be expended on utilities, food, online education and childcare leading to less anxiety. This agrees with Nicola et al. (2020) and Coibion et al. (2020) who reported the socio-economic implications of the pandemic.

Respondents complied with the use of nose/face masks, stay at home policy, social distancing and regular hand hygiene as $84.6 \%$ and $89.4 \%$ used hand sanitizers, and soap and water respectively. Majority of the respondents rarely left their houses during the lockdown (84.6 
$\%)$, avoiding crowded places and physical contact. This suggests that Nigerians are generally very cautious. Health authorities should continue providing education and outreach materials, to increase public understanding of the disease.

Interestingly, of the 200 married respondents, $12.8 \%$ reported that their sexual performance was at its highest frequency apparently due to greater time spent with their spouses. However, $46.7 \%$ reported that frequency of sexual performance was same as before the pandemic, a situation that might be linked to shrewd time management while indoor. Indoor activities including doing office work at home take the mind off sexual intercourse. Sexual desire and frequency of intercourse were also reported to have significantly increased during the COVID19 pandemic in Turkey and Italy, whereas quality of sexual life significantly decreased (Tuskel \& Ozgor, 2020; Cocci, 2020). About $9.7 \%$ of the respondents reported it was at its lowest ever. This may be due to phobia for unplanned pregnancy, anxiety over the pandemic which affects libido and deficient female contraception leading to preference for sexual abstinence.

Also worthy of note is the fact that hospital/clinic visits for non-COVID related cases was low $(10.6 \%)$. This could lead to premature deaths from cases such as trauma, hypertension, childbirth etc. This may be due to the stigma associated with COVID-19 and the risk of infection or wrong diagnosis in hospitals. Stigma towards COVID-19 is caused by fear of its mortality and high communicability (Abdelhafiz et al., 2020). This can be resolved through proper education and transparency of healthcare policies.

A large percentage of respondents $(85.3 \%)$ hold the view that the COVID-19 is a man-made biological weapon. This was also reported by Olapegba et al. (2020). It is important that the Nigerian government and other stake holders embark on campaigns to raise awareness of the true sources of COVID-19 to curb prejudice against foreign nationals. 
Majority viewed the lockdown instituted by the government as necessary $(81.5 \%)$ and agree that the virus can be successfully controlled by the Nigerian government if more efforts are put in contact tracing. This can be explained by the government's unprecedented actions and prompt response in taking stringent control and precautionary measures against previous epidemics, to safeguard the well-being of its citizens. This finding is consistent with recent studies conducted in China and Saudi Arabia, where majority of the participants were convinced that the disease is curable and that their country will combat the disease (Zhong et al., 2020; Al-Hanawi et al., 2020). However, these results contrast with findings in the USA that suggest people tend to express negative emotions, during a pandemic that could affect their attitude (Blendon et al., 2004).

This study had its limitations as the survey was done during the lockdown. The adoption of the chain referral sampling technique employed might limit generalizability of the finding to the general population. Also, response rate was low which may be associated with lack of access to internet facilities especially by members of staff in the lower category who may not have smartphones, cost of internet data or incompetence in filling online surveys. Also, data used in the analysis of this study were self-reported, which might suffer from reporting bias. Nevertheless, this study provides a baseline information on the KAP of members of staff of the university community about the ravaging COVID-19 pandemic in Nigeria.

We recommend that 1) The media should be intensively used by governmental and nongovernmental agencies to provide appropriate health education to avert the mounting myths and misinformation about the pandemic and teach the appropriate hygiene measures to adopt.

2) Government should liaise more with national researchers and international organizations to strengthen capacity in the areas of research funding and health care systems to improve testing capacity and contact tracing. 3) A coherent, coordinated and credible policy response would provide the best chance at limiting the economic fallout due to COVID-19. 
In conclusion, this study was significant as it is the first survey to assess the KAP about COVID-19 among this selected population of Nigerians. Knowledge of the disease is considered the stepping-stone to any health education activity that is implemented. All stake holders should intensify their effort in expediting and implementing preventive/control measures against COVID-19 at local, regional, national and international levels.

\section{Authors' contributions}

OTA conceptualised the project and drafted the manuscript, OTA, OOA, AAO and NOAB designed the questionnaire and collected data, OOA, NOAB and POA did the statistical analysis, AKA revised the manuscript critically for important intellectual content. All authors read and approved the final version of the manuscript to be published and agreed to be accountable for all aspects of the work.

\section{Conflict of interest}

All authors declare no conflict of interest.

\section{Financial support}

None.

\section{Acknowledgement}

The authors are grateful to the management, executives of Associations, deans of Colleges and directors of Directorates of FUNAAB. All the respondents are also acknowledged.

\section{References}

Abdelhafiz, A. S., Mohammed, Z., Ibrahim, M. E., Ziady, H. H., Alorabi, M., Ayyad, M., \& Sultan, E. A. (2020). Knowledge, perceptions, and attitude of egyptians towards the novel coronavirus disease (COVID-19). Journal of Community Health, 1-10. https://doi.org/10.1007/s10900-020-00827-7 
Al-Hanawi, M. K., Angawi, K., Alshareef, N., Qattan, A. M. N., Helmy, H. Z., Abudawood, Y., Alqurashi, M., Kattan, W. M., Kadasah, N. A., Chirwa, G. C., \& Alsharqi, O. (2020). Knowledge, attitude and practice toward COVID-19 among the public in the Kingdom of Saudi Arabia: A cross-sectional study. Frontiers in Public Health, 8, 217. doi: 10:3389/fpubh.2020.00217

Berhan, Y. (2020). Will Africa be devastated by Covid-19 as many predicted? Perspective and Prospective. Ethiopian Journal of Health Sciences, 30, 459-467. http://dx.doi.org/10.4314/ejhs.v30i3.17

Blendon, R. J., Benson, J. M., DesRoches, C. M., Raleigh, E., \& Taylor-Clark, K. (2004). The public's response to severe acute respiratory syndrome in Toronto and the United States. Clinical Infectious Diseases, 38, 925-931. https://doi.org/10.1086/382355

Brug, J., Aro, A. R., Oenema, A., de Zwart, O., Richardus, J. H., \& Bishop, G. D. (2004). SARS risk perception, knowledge, precautions and information sources, the Netherlands. Emerging Infectious Diseases, 10, 1486-1489. doi: 10.3201/eid1008.040283

Choi, J. S., \& Yang, N. Y. (2010). Perceived knowledge, attitude, and compliance with preventive behavior on influenza A (H1N1) by university students. Journal of Korean Academy of Adult Nursing, 22, 250-259.

Cocci, A., Giunti, D., Tonioni, C., Cacciamani, G., Tellini, R., Polloni, G., Cito, G., Presicce, F., Di Mauro, M., Mineruini, A., \& Cimino, S. (2020). Love at the time of the Covid-19 pandemic: Preliminary results of an online survey conducted during the quarantine in Italy. International Journal of Impotence Research, 1-2. https://doi.org/10.1038/s41443-0200305-x 
Coibion, O., Gorodnichenko, Y., \& Weber, M. (2020). The cost of the covid-19 crisis: Lockdowns, macroeconomic expectations, and consumer spending (No. w27141). National Bureau of Economic Research. http://www.nber.org/papers/w27141

Fiorillo, A., \& Gorwood, P. (2020). The consequences of the COVID-19 pandemic on mental health and implications for clinical practice. European Psychiatry, 63, 12. https://doi.org/10.1192/j.eurpsy.2020.35

Geldsetzer, P. (2020). Use of rapid online surveys to assess people's perceptions during infectious disease outbreaks: a cross-sectional survey on COVID-19. Journal of Medical Internet Research, 22(4), e18790. doi: 10.2196/18790

Goumenou, M., Spandidos, D. A., \& Tsatsakis, A. (2020). Possibility of transmission through dogs being a contributing factor to the extreme Covid-19 outbreak in North Italy. Molecular Medicine Reports, 21(6), 2293-2295. https://doi.org/10.3892/mmr.2020.11037

Huang, C., Wang, Y., Li, X., Ren, L., Zhao, J., Hu, Y., Zhang, L., Fan, G., Xu, J., Gu, X., Cheng, Z., Yu, T., Xia, J., Wei, Y., Wu, W., Xei, X., Yin, W., Li, H., \& Cao, B. (2020). Clinical features of patients infected with 2019 novel coronavirus in Wuhan, China. The Lancet, 395, 497-506. https://doi.org/10.1016/S0140-6736(20)30183-5

Hussain, Z. A., Hussain, S. A., \& Hussain, F. A. (2012). Medical students' knowledge, perceptions, and behavioral intentions towards the H1N1 influenza, swine flu in Pakistan: a brief report. American Journal of Infection Control, 40, e11-e13. https://doi.org/10.1016/j.ajic.2011.12.004

Isere, E. E., Fatiregun, A. A., \& Ajayi, I. O. (2015). An overview of disease surveillance and notification system in Nigeria and the roles of clinicians in disease outbreak prevention and control. Nigerian Medical Journal, 56, 161-168. doi: 10.4103/0300-1652.160347 
Izah, S. C., Iniamagha, I., \& Sylva, L. (2020). Two months of COVID-19 pandemic in Nigeria: Distribution and responses of the Nigerian media. Epidemiology International, 5, 32-44. https://doi.org/10.24321/2455.7048.202015

Lau, J. T. F., Yang, X., Tsui, H., \& Kim, J. H. (2003). Monitoring community responses to the SARS epidemic in Hong Kong: from day 10 to day 62. Journal of Epidemiology and Community Health, 57, 864-870. http://dx.doi.org/10.1136/jech.57.11.864

Li, C., Yang, Y., \& Ren, L. (2020). Genetic evolution analysis of 2019 novel coronavirus and coronavirus from other species. Infection, Genetics and Evolution, 82, 104285. https://doi.org/10.1016/j.meegid.2020.104285

Morganstein, J. C., \& Ursano, R. J. (2020). Ecological disasters and mental health: causes, consequences, and interventions. Frontiers in Psychiatry, 11, 1. https://doi.org/10.3389/fpsyt.2020.00001

Nicola, M., Alsafi, Z., Sohrabi, C., Kerwan, A., Al-Jabir, A., Iosifidis, C., Agha, M., \& Agha, R. (2020). The socio-economic implications of the coronavirus and COVID-19 pandemic: a review. International Journal of $\quad$ Surgery, $\quad 78, \quad 185-193$. https://doi.org/10.1016/j.ijsu.2020.04.018

Nigeria Centre for Disease Control (2020, June 13). An update of COVID-19 outbreak in Nigeria for Week 24. Retrieved from www.covid19.ncdc.go.ng

Olapegba, P. O., Ayandele, S., Kolawole, S. O, Oguntayo, R., Gandi, J. C., Dangiwa, A. L., Ottu, I. F. A., \& Iorfa, S. K. (2020). A preliminary assessment of novel Coronavirus (COVID19) knowledge and perceptions in Nigeria. Social Sciences and Humanities Open. http://dx.doi.org/10.2139/ssrn.3584408 
Olaseni, A. O., Akinsola, O. S., Agberotimi, S. F., \& Oguntayo, R. (2020). Psychological distress experiences of Nigerians amid COVID-19 pandemic. Social Sciences and Humanities Open. SSHO-D-20-00416. http://dx.doi.org/10.2139/ssrn.3596106

Otitoloju, A. A., Okafor, I. P., Fasona, M., Bawa-Allah, K. A., Isanbor, C., Onyeka, C. S., Folarin, O. S., Adubi, T. O., Sogbanmu, T. O., \& Ogbeibu, A. E. (2020). COVID-19 pandemic: examining the faces of spatial differences in the morbidity and mortality in sub-Saharan Africa, Europe and USA. MedRxiv. https://doi.org/10.1101/2020.04.20.20072322

Richards, P. (2017). Ebola: How a people's science helped end an epidemic. London: Zed Books.

Roy, D., Tripathy, S., Kar, S. K., Sharma, N., Verma, S. K., \& Kaushal, V. (2020). Study of knowledge, attitude, anxiety and perceived mental healthcare need in Indian population during COVID-19 pandemic. Asian Journal of 102083. https://doi.org/10.1016/j.ajp.2020.102083

Sohrabi, C., Alsafi, Z., O’Neill, N., Khan, M., Kerwan, A., Al-Jabir, A., Iosifidis, C., \& Agha, R. (2020). World Health Organization declares global emergency: A review of the 2019 novel coronavirus (COVID-19). International Journal of Surgery, 76, 71-76. https://doi.org/10.1016/j.ijsu.2020.02.034

Tang, X., Wu, C., Li, X., Song, Y., Yao, X., Wu, X., Duan, Y., Zhang, H., Wang, Y., Qian, Z., Cui, J., \& Lu, J. (2020). On the origin and continuing evolution of SARS-CoV-2. National Science Review. doi: 10.1093/nsr/nwaa036

Vartti, A. M., Oenema, A., Schreck, M., Uutela, A., de Zwart, O., Brug, J., \& Aro, A.R. (2009). SARS knowledge, perceptions, and behaviors: A comparison between Finns and the Dutch during the SARS outbreak in 2003. International Journal of Behavioral Medicine, 16, 41-48. doi: 10.1007/s12529-008-9004-6 
Voeten, H., de Zwart, O., Veldhuijzen, I. K., Yuen, C., Jiang, X., Elam, G., Abraham T., \& Brug, J. (2009). Sources of information and health beliefs related to SARS and Avian influenza among Chinese communities in the United Kingdom and The Netherlands, compared to the general population in these countries. International Journal of Behavioral Medicine, 16, 49-57. doi: 10.1007/s12529-008-9006-4

World Health Organisation (2020, June 13). Coronavirus disease (COVID-19) Situation Report - 144. Retrieved from www.who.int/covid-19/information

Yuksel, B., \& Ozgor, F. (2020). Effect of the COVID-19 pandemic on female sexual behavior. International Journal of Gynecology and Obstetrics. https://doi.org/10.1002/ijgo.13193

Zetzsche, D.A. (2020). One million or one hundred million casualties? The impact of the COVID-19 crisis on low-and middle-income countries. Social Sciences and Humanities Open. http://dx.doi.org/10.2139/ssrn.3597657

Zhong, B. L., Luo, W., Li, H. M., Zhang, Q. Q., Liu, X. G., Li, W. T., \& Li, Y. (2020). Knowledge, attitudes, and practices towards COVID-19 among Chinese residents during the rapid rise period of the COVID-19 outbreak: A quick online cross-sectional survey. International Journal of Biological Sciences, 16, 1745-1752. doi: 10.7150/ijbs.45221 